\title{
Identification of the most critical pipes in the presence of imprecise information
}

\author{
M. Compare \\ Energy Department, Politecnico di Milano, Via La Masa 34, 20133 Milano, Italy
}

Aramis Srl, Via Pergolesi 5, 20124, Milano, Italy

A. Mancuso

Energy Department, Politecnico di Milano, Via La Masa 34, 20133 Milano, Italy

T. Laakso

Department of Civil and Environmental Engineering, Aalto University, Tietotie 1 E, Espoo, Finland

A. Salo

Systems Analysis Laboratory, Department of Mathematics and Systems Analysis, Aalto University School of Science, Otakaari 1, Espoo, Finland

E. Zio

Energy Department, Politecnico di Milano, Via La Masa 34, 20133 Milano, Italy

Chair on Systems Science and the Energetic Challenge, European Foundation for New Energy-Electricité de France, Ecole Centrale Paris and Supelec, France

Aramis Srl, Via pergolesi 5, Milano, Italy

ABSTRACT: Risk-Based Maintenance (RBM) helps focus maintenance efforts on the more risky items of the asset under study. In particular, RBM can be applied to maintain large infrastructure networks whose preventive maintenance programmes are a major determinant of the operating costs of the network and its competitiveness. In this paper, we propose a novel risk-based methodology to select the most risky items of the network, based on qualitative expert statements about risk-related attributes and weights which characterize the relative importance of these risks. The methodology is illustrated with a real case study on a large sewerage network in Espoo, Finland.

\section{INTRODUCTION}

Large infrastructure networks, such as gas or water pipeline systems, undergo preventive maintenance programmes that are a significant part of network operating costs (Zhao, 1998), (Zhao \& Rajani, 2002). Maintenance optimization is fundamental to the effective management of these complex assets. Information about the current condition of assets is needed for maintenance optimization; but there is a challenge in that typically only rough estimates about the actual pipe deterioration profiles is available, particularly in the case of large and buried infrastructure networks which are costly to inspect.

In this situation, maintenance planning can be framed as a process which involves sub-problems:

i) Identification of an optimal set (portfolio) of pipe inspections for which the subsequent renovation actions (if necessary) can be expected to improve the network operability most while also enabling the largest cost saving.

ii) Once the degradation states of the selected pipes have been assessed through inspections, identify an optimal plan for maintenance actions on the whole network.

In this paper, we propose a novel risk-based methodology for selecting the most critical pipes of a net- work, which is fundamental for addressing sub-problem i). The identification of optimal portfolios of inspections and maintenance actions upon inspections will be tackled in future research.

Our risk-based methodology has been developed in collaboration with the Department of Civil and Environmental Engineering at Aalto University. It makes it possible to accommodate imprecise statements that experts provide about the risk associated with network pipes. The methodology is generic in that it can be applied to different types of network (gas, water, wastewater, etc.). We illustrate it with a real application to the analysis of a large sewerage network in Espoo, Finland.

\section{NETWORK ANALYSIS}

In Helsinki region, water and wastewater services are provided by the Helsinki Region Environmental Services Authority (HSY) which serves about one million customers.

In order to manage the network effectively, HSY has built a database which contains following information about every pipe: 
- Pipe features: The ID code, installation year, location (in terms of xyz-coordinates for both endpoints), diameter, depth, type (gravitational or pressure sewer), possible renovation year (in case the pipe has been renovated), and material. The most common pipe material is concrete, but some pipes are made of cast iron, polyethylene, PVC or renovated by felt socks.

- Inspection results: The possible inspection year and outcome. For each inspected pipe, the inspection result is stored in the database, together with the location and type of each individual fault that has been detected during inspections (e.g. slump, hole, tree roots, pile-up).

- Maintenance history: The number of blockages and flushing events is collected in the DB.

- Pipe external context: Other significant information related to the surrounding environment in which the pipe is located (i.e., buildings, traffic load, groundwater areas, and soil type). Most pipes are in clayish soil, but some are located in moraine, bedrock, organic, coarse silt or sandy soils.

The case study considered in this paper is based on a subset of $J=6103$ pipes of the sewerage network in Espoo, Finland. This network is made up of more than 33000 pipes, and has a total length of about $900 \mathrm{~km}$.

\section{METHODOLOGY SNAPSHOT}

An accurate risk analysis is the basis of the riskbased maintenance approach (Modarres, 2006). In this respect, although there exist several definitions of risk (e.g., see Aven \& Renn, 2009 and Aven, 2012 for reviews and comparisons), in maintenance engineering risk has always been viewed as a combination of two failure attributes: likelihood (i.e., description of the uncertainty with which the failure is expected to occur) and severity (i.e., a quantification of the impact of failure on dimensions such as environment, safety, production) (Kaplan, 1981).

In the case of large underground networks, the risk of the pipe failures cannot usually be precisely assessed, because there is merely imprecise knowledge and/or uncertain information about $i$ ) the condition of the pipes (which are costly to inspect) and ii) the impact of pipe failures on concerns such as the built environment and safety, among others.

The RBM methodology proposed in this paper builds on the Multi Attribute Value Theory (MAVT, e.g., French, 1988 and Keeney \& Raiffa, 1976) to define the risk associated to the pipes, based on qualitative statements provided by experts. In short, MAVT is a systematic approach for tackling decision problems which involve multiple objectives.

Within the proposed MAVT-based framework, the problem of assigning risk priorities to the pipes of the network has two main objectives:
1. Identify pipes which are most likely to fail.

2. Identify pipes whose failure has the most se-

vere consequences.

For each objective, a team of experts analyzes which available indicators affect, according to the experience of these experts, failure likelihood and severity, respectively. These indicators are structured as a hierarchy which provides decision support by decomposing the overall objective appropriately into more controllable factors (Keeney \& Raiffa, 1976), (Saaty, 1990). In particular, the hierarchy provides the DM with an overall view of the complex relationships inherent in the decision problem, and helps him/her assess whether the objectives or criteria at each level are of the same order of magnitude so that they can be meaningfully compared with each other.

For example, to meet the objective of identifying pipes with the highest failure likelihoods, the decision maker estimates which sub-indicators (such as pipe age and material) matter most (Figure 1). The decomposition proceeds until no further refinements are needed. The sub-indicators at the lowest level of the hierarchy are called leaves, and for every leaf we need to specify appropriate values that the alternatives can attain on these sub-indicators. For example, the indicator "Material" (where the objective is to find pipes with the weakest material) can assume one out of the following values 'PVC', 'cast iron', 'concrete', 'polyethylene', 'renovated by felt socks'.

If the leaf indicators are mutually preferentially independent (Keeney \& Raiffa, 1976), an additive function can be used to calculate the overall value $j$ of any alternative (i.e., pipe) $x^{j}, j=1, \ldots, J$

$V\left(x^{j}\right)=\sum_{i=1}^{n} w_{i} \cdot v_{i}\left(x_{i}^{j}\right)$

where $n$ is the number of leaf indicators, $w_{i}$ is the importance of the $i$-th leaf indicator with respect to the others, $x_{i}^{j}$ is the value (or consequence) of indicator $i$ for alternative $x^{j}$, and $v_{i}\left(x_{i}^{j}\right)$ represents its rating. More specifically, at every level $l_{h}$ in the hierarchical structure, the experts express preferences to establish the relative impact of the sub-indicators (at level $l_{h+1}$ ) on the corresponding indicator at the higher level $l_{h}$. These preference statements are translated into possibly uncertain values of indicator weights at level $l_{h+1}$. Then, the weights at every hierarchical level are propagated through the tree to yield the weights $w_{i}$ of the leaf attributes (Keeney \& Raiffa, 1976), (Punkka \& Salo, 2013), (Saaty, 1990), (Salo \& Hämäläinen, 1995). In our approach, the DM's preferences are elicited by applying the extension (Salo \& Hämäläinen, 1995) of the PAIRS method (Salo \& Hämäläinen, 1992), which makes it possible to admit imprecise preference statements such as 'objective $i$ is more important than objective $i+1$ '. 
Ratings in the range $[0,100]$ are assigned to $x_{i}^{j}, i=$ $1, \ldots, n, j=1, \ldots, J$ through the SWING procedure (Von Winterfeldt \& Edwards, 1986). Finally, combining these ratings with the weights of the sub-indicators gives a full ranking for the alternatives.

Note that the procedure of associating the corresponding rate vector with each pipe can be easily automated, because the measurement values of the leaf indicators are pipe characteristics such as material and age, characteristics of the location of the pipe. These are stored in the databases maintained by the network utilities so that a vector of leaf measurement values can be automatically assigned to every pipe. The corresponding rates come from the SWING procedure, which is performed once for every leaf sub-indicator (except updating).

The above procedure assigns two overall values to each pipe: the likelihood value and the severity value. On this basis, the pipes can be compared with each other to identify those that are characterized by the highest failure likelihood and the highest failure severity. In this respect, given the uncertainty in both the likelihood and severity values, the pairwise dominance concept (Salo \& Hämäläinen, 1995) is utilized to select the non-dominated pipes (i.e., the Pareto optimal set) in the two-dimensional space defined by failure likelihood and failure severity.

\section{LIKELIHOOD AND SEVERITY}

\subsection{Likelihood}

Figure 1 shows the hierarchical structure of failure likelihood that was identified based on expert interviews. The sub-indicators at level $l_{2}$ are:

- Pipe features: In order to identify the pipes with the highest failure likelihood, one can evaluate the importance of pipe-specific characteristics such as the pipe construction material, age since last renovation and diameter. Note that we are not considering the pipe installation year attribute, because in our dataset this is strongly correlated with (and thus captured by) the pipe material type.

- Past events: The larger the number of past blockages and flushing, the larger the probability of disruptions in the near future.

- Local circumstances: The elements of the surrounding environment can contribute significantly to the failure likelihood. According to the experts' opinion, the most important factors are the soil and the traffic load.

The weights were elicited with the PAIRS method (Salo \& Hämäläinen, 1992). That is, for every indicator at the second level, $l_{2}$, of the hierarchical tree, the sub-indicators are ranked based on their importance by maximizing the failure likelihood. In our case study, the increase in the harshness of local surround- ings was judged to be the least important sub-indicator from the perspective of maximizing the failure likelihood, with no preference between pipe features and past events. These statements correspond to the following inequalities

$$
\begin{gathered}
w_{\text {pipe features }} \geq w_{\text {local circumstances }} \\
w_{\text {past events }} \geq w_{\text {local circumstances }}
\end{gathered}
$$

which, together with the constraint that the weights have to sum to 1 , define the region where the actual, but unknown, triplet of weights is located.

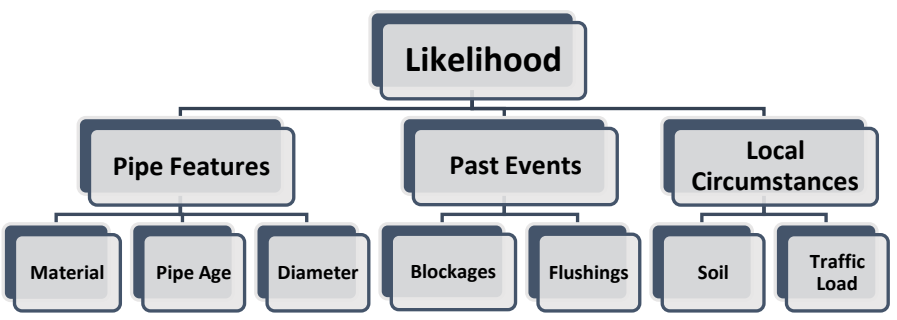

Figure 1: The hierarchy of indicators for failure likelihood.

With regard to the importance of the indicators at level $l_{3}$ of the tree with respect to those at level $l_{2}$, the experts stated that:

- Pipe diameter is the most important sub-indicator among those of the pipe features-the smaller the diameter, the higher the failure likelihood.

- The number of past blockages is more important than the number of past flushing.

- Soil is equally important as traffic load.

\subsection{Severity of Consequences}

The consequence tree is a hierarchical representation of the attributes which define how severe impacts pipe failures have on properties, environment, and safety and how possible network malfunctions affect water consumers. The methodology for assigning a likelihood value to the pipes could also have been adopted to evaluate the severity values. However, in this study, we use recent results from the Department of Civil and Environmental Engineering at Aalto University in which consequences have been evaluated by mainly taking account of pipe location and surroundings, and the pipe specific flow (Laakso et al., 2015).

A severity value can be assigned to each pipe based on the criteria in Table 1. Namely, the pipe belongs to Class 1 only if it meets at least one of the criteria 1-8. Otherwise, one checks if the pipe meets one out of the criteria 9-19 for being of Class 2. The other pipes are assigned to the third criticality class.

Finally, note that if the analysis were to be based on the severity tree only, then inspections would focus on the pipes whose position is more hazardous, independently on their expected current degradation state. This highlights the added value of prioritizing 
the inspections by taking account of both failure likelihood and severity.

Table 1: Criteria used for defining the pipe-specific criticality classification for sewer pipes.

\begin{tabular}{|c|c|}
\hline & Class 1 \\
\hline 1 & $\begin{array}{l}\text { Sewer mains of crucial functional importance for } \\
\text { the whole network }\end{array}$ \\
\hline 2 & Main tunnels \\
\hline 3 & $\begin{array}{l}\text { Sewer mains and pressure sewers that are within } \\
\text { significant groundwater areas }\end{array}$ \\
\hline 4 & $\begin{array}{l}\text { Sewers close to primary or secondary raw water re- } \\
\text { sources }\end{array}$ \\
\hline 5 & Pipes under railways \\
\hline 6 & Pipes under significant roads \\
\hline 7 & $\begin{array}{l}\text { Undoubled pressure pipes from critical pump sta- } \\
\text { tions }\end{array}$ \\
\hline \multirow[t]{2}{*}{8} & Very high pipe-specific flow \\
\hline & Class 2 \\
\hline 9 & Main sewer not included in Class 1 \\
\hline 10 & $\begin{array}{l}\text { Sewers in protected areas / nature conservation ar- } \\
\text { eas }\end{array}$ \\
\hline 11 & Pipes crossing main water tunnels \\
\hline 12 & Pipes going under a water body (river, lake, sea) \\
\hline 13 & Pipes under buildings \\
\hline 14 & Pipes close to protected ditches \\
\hline 15 & Pipes close to swimming beaches \\
\hline 16 & $\begin{array}{l}\text { Pipes other than sewer mains which are within sig- } \\
\text { nificant groundwater areas }\end{array}$ \\
\hline 17 & $\begin{array}{l}\text { Sewer mains within groundwater areas of less sig- } \\
\text { nificance }\end{array}$ \\
\hline 18 & $\begin{array}{l}\text { Sewers close to critical underground structures } \\
\text { (e.g. subway) }\end{array}$ \\
\hline \multirow[t]{2}{*}{19} & High pipe-specific flow \\
\hline & Class 3 \\
\hline 20 & Every remaining pipe \\
\hline
\end{tabular}

\section{RATING ELICITATION}

As mentioned above, the SWING approach (Von Winterfeldt \& Edwards, 1986) was applied to assign the ratings to the indicator values of each leaf. Namely, for each indicator the value that increases the failure likelihood or severity the most, was assigned the rate 100 and, with a similar procedure, the indicator with the lowest impact on failure likelihood or severity was assigned the rate 0 . For example, when considering the leaf attribute 'material quality' in the failure likelihood tree, the least reliable material is PVC, while the most reliable is concrete. These were thefore assigned ratings 100 and 0 , respectively.

In the next elicitation questions, the respondents were asked to state their ordinal preferences for quality differences. Specifically, the decision maker was asked which 'swing' in the indicator value to the best one would result in the largest, the second largest, etc., improvement. Again with reference to the material quality attribute, the answers to these questions have led to the following ranking in ascending order: 'concrete', 'polyethylene', 'cast iron', 'renovated by felt socks' and 'PVC'.

Finally, the intermediate quality classes were evaluated with respect to the extreme values and, for validation, with respect to each other. For example, one of the questions for the material attribute was the following: "Is the quality difference between cast iron and concrete pipes more or less significant than that between PVC and cast iron pipes?". Criticality of cast iron pipes is closer to concrete than PVC pipes; therefore, its uncertain score is closer to 0 (concrete criticality score) than 100 (PVC criticality score).

This way, experts were able to assign interval scores to each class according to the proximity to one of these conditions and the other elicited interval scores. The responses were recorded into an Excel tool, and they were visually adjusted and validated as well.

By this procedure, experts assigned an interval cardinal score in the range of 0-100 to each leaf indicator class of the failure likelihood tree.

Failure severity was evaluated for each pipe according to the criteria in Table 1; these criteria were first ordered according to their severity. For example, a pipe disruption close to a railway is a more severe situation than a pipe failure near a beach. For this reason, the interval score of the former pipe is larger than that of the latter.

These criteria were assigned uncertain ratings by applying the SWING procedure. Namely, score 0 was assigned to pipes of class 3, whereas 100 to the most critical circumstance in Class 1 (no. 8: "Very high specific flow"). The remaining 18 intermediate severity conditions were assessed by eliciting imprecise score intervals by comparing with the two extreme conditions and the other elicited ratings. This way, experts identified interval cardinal scores in the range of 0-100 for each intermediate circumstances based on their criticality level. Similarly to the principles applied in (Laakso et al., 2015), we assumed that if a pipe met more than one condition, then it had the highest score among those corresponding to the met conditions.

\section{ALTERNATIVE OVERALL VALUE}

The recursive algorithm presented in Salo \& Hämäläinen, 1992 was adopted to compute the overall score intervals of failure likelihood $V_{L}\left(x^{j}\right)=$ $\left[v_{L}\left(x^{j}\right), \bar{v}_{L}\left(x^{j}\right)\right]$ and failure severity $V_{C}\left(x^{j}\right)=$ $\left[\underline{v}_{C}\left(x^{j}\right), \bar{v}_{C}\left(x^{j}\right)\right]$ for each pipe $x^{j}$, where $j=$ $1, \ldots, J$. This algorithm makes it possible to propagate efficiently the uncertainty of the interval-valued ratings of the leaf indicators and the imprecise weights of the sub-objectives through the different levels of 
the hierarchical trees onto the range of possible overall values at the topmost objectives.

\section{RISK ASSESSMENT}

In this Section, we consider the identification of the most critical pipes which belong to the Pareto optimal set in the two dimensional space of failure likelihood and failure severity. Generally speaking, a pipe belongs to the Pareto optimal set if it is not dominated; that is, if its failure likelihood cannot be increased without simultaneously decreasing the severity of the failure consequences and vice versa.

However, this 'traditional' concept of dominance needs to be modified, since the overall values of both likelihood and severity are interval numbers $\left(V_{L}\right.$, and $V_{C}$, respectively) instead of crisp numbers. Thus, with interval scores, pipe $x^{j}$ is said to dominate pipe $x^{t}$ $(t \neq j)$ if and only if the intervals $V_{L}\left(x^{j}\right)$ and $V_{C}\left(x^{j}\right)$ both lie above $V_{L}\left(x^{t}\right)$ and $V_{C}\left(x^{t}\right)$, respectively:

$$
\begin{aligned}
& x^{j}>x^{t} \\
& \leftrightarrow\left\{\begin{array}{l}
\underline{v}_{L}\left(x^{j}\right)>\bar{v}_{L}\left(x^{t}\right) \\
\underline{v}_{C}\left(x^{j}\right) \geq \bar{v}_{C}\left(x^{t}\right)
\end{array}\right\} \vee\left\{\begin{array}{l}
\underline{v}_{L}\left(x^{j}\right) \geq \bar{v}_{L}\left(x^{t}\right) \\
\underline{v}_{C}\left(x^{j}\right)>\bar{v}_{C}\left(x^{t}\right)
\end{array}\right\}
\end{aligned}
$$

This dominance definition allows us to identify the optimal Pareto set $F_{1}$ (Keeney \& Raiffa, 1976), which contains $J^{1}=2079$ non-dominated solutions (circle marker and solid line in Figure 2), out of the $J=6103$ pipes of the network. Then, we remove these solutions from the original pipe dataset and select the $J^{2}$ non-dominated solutions, $F_{2}$, in the remaining set (cross marker and dashed line in Figure 2). This procedure continues until the set of remaining non-dominated solutions is empty. In the case study of the Espoo sewerage system, three Pareto fronts have been identified, the third, $F_{3}$, being marked by squares in Figure 2.

The identification of different Pareto frontiers makes it possible to consider levels of decreasing criticality: pipes belonging to the first Pareto set are the most critical pipes, and the successive analyses will focus on such Pareto set.

\section{CONCLUSIONS}

In this paper, we have developed a risk-based inspection model for a large sewerage network in Espoo, Finland. The risk assessment, based on the two entries failure likelihood and failure severity, makes it possible to identify the most critical pipes from which the optimal portfolios for inspections can be found.

Future research will focus on the development of a methodology to estimate the benefit of inspecting the pipes and, on this basis, the selection of optimal portfolio of inspections taking into account uncertain cost estimates as well as possible synergies among the inspections (e.g., inspecting nearby pipes may cost less than inspecting pipes that are far apart).

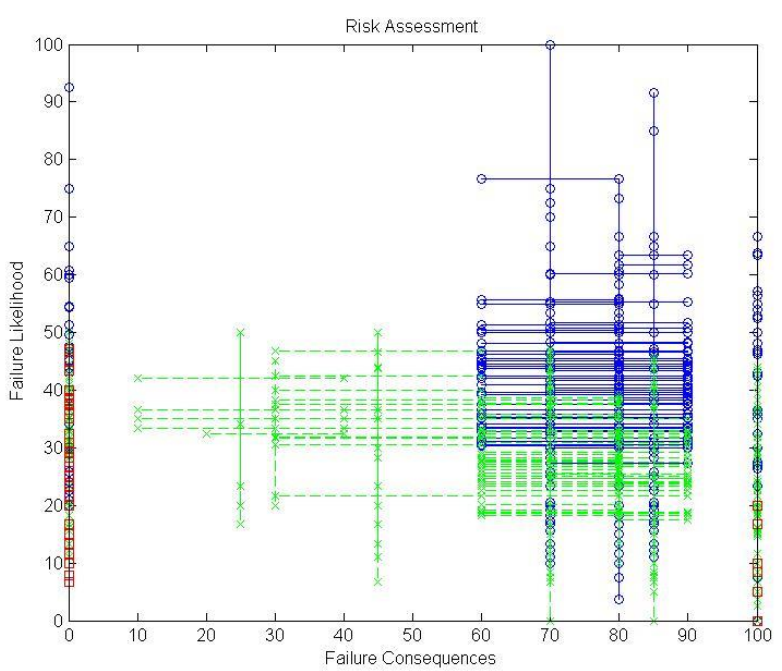

Figure 2: Overall values of failure likelihood and severity of the 6103 pipes considered in the Espoo sewer system.

\section{ACKNOWLEDGMENTS}

This research has been carried out through a close cooperation between the Institutions of the Authors, which are listed in alphabetical order. The Authors wish also to thank the Prof. Riku Vahala's research group at Department of Civil and Environmental Engineering at Aalto University.

\section{REFERENCES}

Aven T., 2012, The risk concept-historical and recent development trends, Reliability Engineering and System Safety, 99: 33-44.

Aven T. \& Renn O., 2009, On risk defined as an event where the outcome is uncertain, Journal of Risk Research, 12: 1-11.

Deb, K. \& al., 2002, A Fast Elitist Non-Dominated Soring Genetic Algorithm for Multi-Objective Optimization: NSGAII, IEEE Transactions on Evolutionary Computation 6 (2): 182-197.

French, S., 1988 , Decision Theory: an introduction to the mathematics of rationality, John Wiley \& Sons, New York.

Kaplan, S., 1981, Garrick B.J., "On the quantitative definition of risk," Risk Analysis, 1: 11-27.

Keeney, R.L. \& Raiffa, H., 1976, Decisions with Multiple Objectives: Preferences and Value Trade-Offs, John Wiley \& Sons, New York.

Laakso, T., Lampola, T., Rantala, J., Kuronen, R, Ahopelto, S. \& Vahala, R., 2015, "Pipe-specific criticality classification as a tool for managing risks related to water and wastewater systems", $2^{\text {nd }}$ New Developments in IT \& Water Conference, Rotterdam, The Netherlands.

Modarres, M., 2006, Risk Analysis in Engineering: Techniques, Tools and Trends, CRC Press.

Punkka, A. \& Salo, A., 2013, Preference programming with incomplete ordinal information, European Journal of Operational Research 231 (1): 141-150. 
Saaty, T.L., 1990, How to make a decision: The Analytic Hierarchy Process, European Journal of Operational Research, 9 (26): 9-26.

Salo, A. \& Hämäläinen, R.P., 1992, Preference assessment by imprecise ratio statements, Operations Research, 40/6: 10531061.

Salo, A. \& Hämäläinen, R.P., 1995, Preference programming through approximate ratio comparisons, European Journal of Operational Research 82 (3): 458-475.

Von Winterfeldt, D. \& Edwards, W., 1986, Decision analysis and behavioral research, Cambridge, UK, Cambridge University Press.

Zhao, J.Q.. 1998. Trunk Sewers in Canada. 1998 APWA International Public Works Congress Seminar Series, American Public Works Association, Las Vegas.

Zhao, J.Q. \& Rajani, B., 2002, Construction and rehabilitation costs for buried pipe with a focus on trenchless technologies, Research Report No. 101, Institute for Research in Construction, National Research Council Canada, Ottawa, ON, Canada. 